\title{
El tema de la pregunta y la elaboración de las respuestas en la detección de mentiras
}

\author{
Flor SÁnchez y Alberto Becerra \\ Universidad Autónoma de Madrid
}

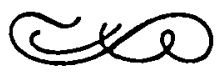

\section{Resumen}

En este trabajo se estudió la incidencia que, sobre la detección de mentiras, podría tener que los temas a que estaban referidas variasen en cuanto a implicación emocional para el emisor y que las respuestas fuesen preparadas o espontáneas. Los resultados obtenidos señalaron que la implicación personal en el tema facilita el reconocimiento de las respuestas falsas, a la vez que la preparación previa de las respuestas dificulta la detección de mentiras a jueces no entrenados.

Palabras clave: Mentira.

\section{The content of questions and the elaboration of answers in deception detection}

\section{Abstract}

In the present study, the effect on deception detection of the emotional message content and previous elaboration of the responses was observed. The results show that personal implication improves the recognition of false responses, and previous elaboration of these answers impairs deception detection by lay judges.

Keywords: Deception.

Agradecimientos: Este trabajo se ha realizado en el marco del proyecto CAYCIT PB85-226.

Dirección del autor: Facultad de Psicología. Universidad Autónoma de Madrid. 28049 Madrid. 
El interés por la detección de la mentira ha generado una larga serie de estudios dirigidos a comparar la habilidad que poseen sujetos no entrenados para discriminar enunciados verdaderos y falsos bajo distintas condiciones experimentales (para una revisión DePaulo y cols., 1980; Zuckerman y cols., 1981).

Una conclusión relevante extraída de estos estudios señala que en contextos de laboratorio, se detectan con mayor precisión los mensajes verdaderos, siendo la detección de mensajes falsos escasamente superior a lo esperado por azar (De Paulo y Rosenthal, 1979). En un intento de explicar este dato se han manejado argumentos que afectan a la conducta de los receptores, a la de los emisores y a los procesos implicados.

Por un lado, los juicios de los sujetos que actúan como detectores de mentira parecen verse afectados por un «sesgo de verdad", pues con más probabilidad evaluan un mensaje como verdadero que como falso cuando no tienen información externa para contrastarlo (Zuckerman y cols., 1984; DePaulo y cols., 1985; McCornack y Parks, 1985), algo similar a lo que ocurre en las situaciones cotidianas (Goffman, 1959).

Por otro lado, las personas que intentan emitir mensajes falsos, hacen una autopresentación de sí mismas que determinará la probabilidad de que sean creídas (Goffman, 1959). Dicha credibilidad estará relacionada con la impresión que consigan transmitir en función de su apariencia física, su estilo expresivo (lenguaje rápido y fluido, mantenimiento del contacto ocular), variables de personalidad (Zuckerman y cols., 1979 Riggio y Friedman, 1983); y habilidades sociales (Riggio y cols., 1987).

El estudio de los procesos cognitivos implicados en la emisión de mentiras, apunta hacia un esfuerzo de procesamiento mayor para elaborar respuestas falsas que para dar respuestas verdaderas, ya que el mensaje falso debe ser construido sin contradecir la información que posea el receptor, sin inconsistencias lógicas y conteniendo detalles suficientes para resultar convincente (Zuckerman y cols., 1981; Cody y cols., 1984). Este sobreesfuerzo en el procesamiento podría modificar los indicadores que acompañan al mensaje (pausas, latencia e ilustradores).

Precisamente, esa consideración de la elaboración del enunciado, tanto de verdad como, muy particularmente de mentira, ha sido uno de los objetivos del presente estudio. En concreto, se pretende indagar la influencia que sobre la precisión en la detección de mensajes verdaderos y falsos puede tener la elaboración previa de la respuesta por parte del emisor. Esta cuestión ha sido abordada en otros estudios, ofreciéndose resultados dispares. Mientras que - para algunos (Littlepage y cols., 1985) la elaboración de las respuestas fsalsas dificulta su detección, para otros (DePaulo y cols., 1983), la facilitaría. La elaboración de la respuesta podría influir el proceso de detección, bien por presentarse indicadores conductuales diferentes para las respuestas espontáneas y planeadas (Kraut, 1978; O'Hair y cols., 1981; Zuckerman y cols., 1981 Cody y O'Hair, 1983; Miller y cols., 1983); como por implicar procesos cognitivos y emocionales diferentes (Greene y cols., 1985). Asímismo, un mensaje más elaborado podría ser más estructurado formalmente, aportar más datos, y resultar más convincente.

La hipótesis que planteamos en relación con la elaboración de la respuesta señala que la oportunidad de preparar una respuesta con anticipación dará lugar a una mentira más plausible, y dada la relación entre la plausibilidad 
de la mentira y las posibilidades de ser creída (Kraut, 1978), las mentiras elaboradas previamente serán menos detectadas que las mentiras espontáneas. Un segundo aspecto planteado por la hipótesis será que las mentiras planeadas serán evaluadas como más creibles que las no planeadas.

Por otra parte, la producción de mensajes falsos implica una actividad emocional de dimensiones complejas (Ekman, 1980: Zuckerman y cols., 1981). Una de ellas se relaciona con el tema respecto al cual los emisores mienten. Bien sea por la naturaleza de su contenido, bien sea por el distinto nivel de implicación personal que para el sujeto tienen los distintos temas (capacidad de generar distintas emociones en el sujeto por el solo hecho de ser emitidas en público, o por el significado especial que para el sujeto tienen), el contenido temático de los mensajes falsos puede, por su implicación emocional, constituirse en una variable que dificulte la construcción del mensaje y por tanto, incidir en la detección de los mismos.

Los temas que se han utilizado en los estudios experimentales han sido tan diversos como los autores que se han interesado por la detección de la mentira, siendo Hocking y cols., (1979) quienes hacen la distinción más precisa al diferenciar entre mentiras sobre-contenidos factuales, aquellas donde el sujeto tiene que mentir en relación a estímulos neutros que le presenta el experimentador y mentiras sobre contenidos emocionales, mediante las cuales el emisor debe describir como agradables sus reacciones emocionales ante los estímulos agradables y desagradables que le son presentados por el experimentador (Ekman y cols., 1974; Hocking y cols., 1979).

En nuestro trabajo los temas sobre los que versaron las respuestas verdaderas y falsas los clasificamos según la implicación personal o emocional que suponía para los sujetos contestar a las preguntas. Relacionamos la implicación emocional con el grado en que al emisor le activa emocionalmente hablar de cada tema en público.

El tema o tópico de la mentira, podría afectar diferencialmente a la emisión y detección de mentiras en la medida en que el sujeto se vea implicado personal o emocionalmente en la pregunta. En el caso de temas con más implicación emocional, a la dificultad diferencial existente entre mentir y decir la verdad, se podría añadir, la de intentar controlar la actividad que puede suponer mentir respecto a cuestiones que generan respuestas emocionales.

En relación a esto nosotros hipotetizamos que los temas con mayor implicación emocional, dado que pueden estar asociados a una mayor activación en el sujeto emisor provocarían expresiones conductuales no controladas, que pueden ser interpretadas por los observadores como indicadores de engaño.

\section{METODO}

\section{Sujetos}

La muestra experimental, la constituyeron 35 estudiantes de $3 .^{\circ}$ de Psicología, con edades comprendidas entre los 20 y 25 años que de forma voluntaria trabajaron con nosotros durante varias semanas.

\section{Variables y Diseño}

Las Variables Independientes del presente estudio dados los objetivos del mismo, fueron las siguientes: 


\section{ELABORACION DE LAS RESPUESTAS}

Esta variable presentaba dos modalidades: Respuestas no elaboradas o espontáneas entendidas como aquellas que el emisor daba inmediatamente después de la formulación de la pregunta y no habiendo tenido conocimiento previo de ésta, y Respuestas elaboradas, aquellas en las que el emisor había preparado la información que daría en público cuando se le formulasen preguntas, que el experimentador le había dado a conocer previamente.

\section{IMPLICACION EMOCIONAL EN LAS RESPUESTAS}

La manipulación en esta variable vino dada por el contenido a que podían estar referidas las preguntas formuladas al emisor por el resto de sus compañeros, quienes habían recibido la consigna de que las preguntas debían estar relacionadas con temas personales y no personales.

La discusión de lo que podía considerarse como tema personal o no personal se hizo en una sesión previa a las sesiones de evaluación, en la que todos los participantes rellenaron un autoinforme en relación a temas sobre los que le importaría hablar o no hablar en público. Algunos de los temas utilizados durante las sesiones fueron: aficiones, animales preferidos, relaciones de pareja, vida sexual, prácticas religiosas, etc.

La operativización de esta variable fue hecha a posteriori por los propios emisores, ya que sólo ellos podían evaluar las preguntas a las que habían respondido como personales o no personales. Así pues, una vez finalizada su intervención, cada emisor categorizó las preguntas que le habían sido formuladas en una escala tipo Likert de 6 puntos, sobre implicación personal en la respuesta. Las puntuaciones obtenidas con esta escala se utilizaron como un indicador de implicación emocional, y fueron utilizadas para analizar posteriormente la influencia de dicha variable en la detección de la mentira.

\section{VALOR DE VERDAD DE LOS ENUNCIADOS}

Esta variable contenía dos categorías ya que las respuestas que daban los emisores podían ser Verdaderas, lo que decían era un hecho comprobable en su vida o. Falsas, lo que decían no se correspondía con su experiencia personal. En el presente estudio el signo de la respuesta fue determinado por los experimentadores conforme a secuencias elegidas al azar.

\section{Variables dependientes:}

Consideraremos una variable dependiente principal que fue la Detección de enunciados verdaderos y falsos, medida por el número de aciertos en el juicio que se valoraron con 1 y errores en el juicio que se valoraron con cero.

Además del juicio sobre el valor de verdad del enunciado, cada evaluador asignaba una puntuación en credibilidad a la respuesta del emisor, para lo cual utilizaba una escala tipo Likert de 6 puntos.

Las variables mencionadas fueron analizadas dentro de un diseño 2(elạboración de las respuestas $=$ espontáneas, elaboradas) X 2(implicación emocional en las respuestas = baja implicación, alta implicación) X 2(valor de verdad de los enunciados $=$ verdad, mentira). 


\section{Procedimiento}

Los datos presentados en este estudio se recopilaron en un seminario sobre la mentira, impartido dentro del calendario de actividades académicas con un carácter teórico-práctico y en el que participaron, de forma totalmente voluntaria, estudiantes que se sintieron atraídos por el tema.

La idea que guió el desarrollo de la investigación obedecía a un intento de romper la artificialidad bajo la cual se han llevado a cabo la mayor parte de las investigaciones en este campo. En estos casos, el procedimiento suele ser que emisor experimental sea filmado de forma individual, mientras responde ante la cámara con sentencias verdaderas o falsas a las preguntas de un experimentador, siendo juzgado posteriormente a través de filmaciones de video.

En nuestro caso pretendímos una situación más natural, que incrementase la validez ecológica del estudio, para lo cual formamos un grupo de trabajo con aquellas personas interesadas en el seminario.

Desde los primeros momentos se planteó una asignación de roles compartida que implicaba que sucesivamente todos participarían bien como receptores o como emisores, planteando la tarea a realizar como un juego donde el éxito estaba en engañar y en no ser engañado, en función del rol que se desempeñase. Para motivarles a la realización de la tarea, se les dio información, recogida de la literatura, sobre las implicaciones que en el desarrollo de su profesión podría tener la habilidad para detectar mensajes falsos.

La disposición de los integrantes del grupo dentro de la sala era en círculo, de tal forma que el sujeto que en cada momento estaba actuando de emisor era observado sin obstáculos por el resto del grupo.

Las personas que actuaron como emisores habían sido informados previamente de que su tarea consistía en responder con sentencias verdaderas o falsas, según le indicara el experimentador, y con el objetivo de no ser detectados cuando mintiesen, a las 10 preguntas que, sucesivamente, le iban a hacer sus compañeros.

La tarea del resto del grupo, que actuaban como decodificadores y evaluadores de las respuestas del emisor, era formular las preguntas y, posteriormente, tras la contestación de éste identificar el valor de verdad o falsedad de sus respuestas.

Las preguntas hechas al emisor estaban guiadas por la curiosidad de sus compañeros por conocer aspectos de su vida (intereses, motivaciones, relaciones), inaccesibles en la interacción cotidiana en clase, y podían ser más o menos personales como ya hemos indicado.

Antes de iniciarse la sesión (formulación de preguntas y evaluación de las respuestas) el grupo, sin la presencia de quién iba a actuar como emisor, decidía las preguntas que deseaba hacerle, siendo anotadas éstas por uno de los experimentadores, quien al azar elegía 4 y se las daba a conocer al emisor aparte, desconociendo el resto del grupo cuáles eran las preguntas que el emisor podría preparar. Mediante un procedimiento aleatorio el experimentador asignaba ante qué preguntas debía emitir o decir la verdad, pudiendo el emisor, elegir la respuesta pero no la condición.

Una vez concluída la preparación de las respuestas, el emisor entraba en la sala y comenzaba la sesión: se le formulaban 10 preguntas, para cuatro de las cuales ya había preparado la respuesta (condición de respuesta elaborada), 
y al resto contestaba según le iban siendo formuladas (condición de respuesta espontánea) tras indicarle el experimentador si la respuesta debía ser verdadera o falsa. Una vez finalizada la respuesta, el grupo juzgaba su veracidad o falsedad y lo anotaban en la hoja de respuestas junto a una puntuación en credibilidad.

La determinación del valor de verdad del enunciado (verdadero o falso) por el exprimentador era un intento de evitar sesgos, impidiendo que el emisor mintiese o dijese la verdad según le fuese más fácil hacer una cosa u otra ante determinadas cuestiones.

Para el análisis de los datos y extracción de los resultados se utilizó el paquete de programas estadísticas BMDP, concretamente el $4 \mathrm{~F}$ para análisis de tablas de frecuencias y modelos lineales logarítmicos.

\section{Resultados}

Los análisis realizados señalan el grado de asociación o independencia entre las condiciones de las distintas variables tomadas individualmente, en combinaciones dos a dos, tres a tres y, por último, la combinación múltiple de las tres variables independientes con la variable criterio: el acierto o error de los juicios emitidos. El análisis pormenorizado de los distintos efectos aportados por todas las combinaciones posibles de factores se detalla a continuación.

Si se observa la Tabla 1, puede comprobarse que, en lo que respecta a los factores principales, todos ellos muestran valores en el test de asociación parcial que son significativos. En realidad, el dato relevante es el que corresponde a la significación de la variable Juicio como efecto principal $X^{2}=59.82 ; \mathrm{p}$ $=.000$. Con este resultado se estaría poniendo de manifiesto que el procedimiento experimental utilizado es discriminativo a la hora de procurar aciertos y errores en los juicios sobre los enunciados (Aciertos $=1396$, Errores $=1016$ ).

Los resultados de las combinaciones dos a dos, muestran efectos secundarios, significativos estadísticamente, tanto en el test de asociación parcial como en el test de asociación marginal. Los que aquí son relevantes, aquéllos que representan una interacción con la variable dependiente acierto o error en el juicio, serían los determinados por la asociación entre esa variable y el Valor de Verdad $\mathrm{X}^{2}$ [asociación parcial] $=12.10, \mathrm{p}=.000 ; \mathrm{X}^{2}$ [asociación marginal $]=11.45, \mathrm{p}=.000 \mathrm{y}$ entre el Juicio y la Elaboración $\left(\mathrm{X}^{2}\right.$ [asociación parcial $]=53.56, \mathrm{p}=0.000 ; \mathrm{X}^{2}$ [asociación marginal $]=53.15, \mathrm{p}=0.000$ ). Asimismo, la interacción entre Juicio e Implicación personal aparece como significativa, si bien con probabilidades asociadas más bajas ( $\mathrm{X}^{2}$ [asociación parcial $]=8.46, \mathrm{p}=.001 ; \mathrm{X}^{2}$ [asociación marginal $\left.]=10.87, \mathrm{p}=.004\right)$. Las interacciones entre la variable Implicación Personal con Elaboración y con Valor de verdad apuntan hacia un posible efecto modulador de aquélla en los resultados alcanzados hasta ahora, mientras que la interacción entre valor de verdad y Elaboración, con una significación, exclusivamente, en el test de asociación parcial viene a incidir en la adecuación del procedimiento experimental utilizado.

En cuanto a los efectos de tercer orden, la Tabla I muestra la significación de la triple interacción Juicio $\mathrm{x}$ Valor de Verdad $\mathrm{x}$ Elaboración, ( $\mathrm{X}^{2}$ [asociación 
parcial] $=15.87, \mathrm{p}=.000 ; \mathrm{X}^{2}$ [asociación marginal] $13.34, \mathrm{p}=.000$ Juicio $\mathrm{x}$ Implicación $\mathrm{x}$ Elaboración $\left(\mathrm{X}^{2}\left[\right.\right.$ asociación parcial] $=23.55, \mathrm{p}=.000 ; \mathrm{X}^{2}$ [asociación marginal] $=26.52, \mathrm{p}=.000$ y Valor de Verdad $\mathrm{x}$ Elaboración $\mathrm{X}$ Implicación $\left(\mathrm{X}^{2}\right.$ [asociación parcial] $=74.04, \mathrm{p}=.000 ; \mathrm{X}^{2}$ [asociación marginal $]=72.47, \mathrm{p}=.000$. Dichos valores son reflejo de la mutua interrelación 0 dependencia entre las variables tratadas.

\section{TABLA I}

Modelo Linear logarítmico. Efecto de las variables Juicio (J) Valor de verdad de los enunciados (V) Implicación emocional (I) Elaboración de las respuestas (E)

\begin{tabular}{|c|c|c|c|c|c|}
\hline \multirow[b]{2}{*}{ Efecto } & \multirow[b]{2}{*}{ G.L. } & \multicolumn{2}{|c|}{ Asoc. Parcial } & \multicolumn{2}{|c|}{ Asoc. Marginal } \\
\hline & & $\mathrm{X}^{2}$ & Probab. & $\mathrm{X}^{2}$ & Probab. \\
\hline $\mathrm{J}$. & 1 & 59.82 & 0.0000 & & \\
\hline V. & 1 & 57.31 & 0.0000 & & \\
\hline I. & 2 & 204.68 & 0.0000 & & \\
\hline $\mathrm{E}$ & 1 & 690.50 & 0.0000 & & \\
\hline JV. & 1 & 12.10 & 0.0005 & 11.45 & 0.0007 \\
\hline JI. & 2 & 8.46 & 0.0145 & 10.87 & 0.0044 \\
\hline JE. & 1 & 53.56 & 0.0000 & 53.15 & 0.0000 \\
\hline VI. & 2 & 84.10 & 0.0000 & 81.90 & 0.0000 \\
\hline VE. & 1 & 5.95 & 0.0147 & 0.94 & 0.3322 \\
\hline IE. & 2 & 28.96 & 0.0000 & 27.00 & 0.0000 \\
\hline IVI. & 2 & 1.05 & 0.5914 & 2.02 & 0.3642 \\
\hline JVE. & 1 & 15.87 & 0.0001 & 13.54 & 0.0003 \\
\hline JIE. & 2 & 23.55 & 0.0000 & 26.52 & 0.0000 \\
\hline VIE. & 2 & 74.04 & 0.0000 & 72.47 & 0.0000 \\
\hline JVIE. & 2 & 0.99 & 0.6088 & & \\
\hline
\end{tabular}

En la Tabla II, el análisis de los aciertos y errores respecto a enunciados Verdaderos (aciertos $=631$, errores $=389$ ) primera condición de la variable Valor de Verdad, muestra un patrón prácticamente idéntico al obtenido anteriormente, con la única salvedad de que, cuando de estos enunciados se trata, no aparece una asociación entre el acierto o error en el Juicio y la implicación personal del emisor en el enunciado expresado.

Cuando se analizan los enunciados Falsos (aciertos $=765$, errores $=627$ ), resultados que aparecen en la Tabla III, es de nuevo, la similitud con respecto al caso general la característica más llamativa. En este caso es la interacción Implicación por Elaboración la que no resulta significativa.

No obstante la significación de las interacciones múltiples $\left(X^{2}=10.28\right.$, $\mathrm{p}=.005)$ para las respuestas verdaderas y $\left(\mathrm{X}^{2}=14.25\right)$ para las falsas vendría a subsanar la no aparición de las significaciones comentadas.

En síntesis, los resultados alcanzados mediante los análisis realizados señalan, en primer lugar, una asociación entre el acierto de la evaluación por parte de los receptores con el nivel de elaboración del enunciado. A este respecto, hipotetizamos una influencia en la detección de la mentira evaluada a través del número de aciertos y fallos cometidos. Los resultados corroboran nuestra aproximación y, así, la elaboración de la respuesta disminuye la precisión de los aciertos en el juicio (respuestas no elaboradas: aciertos = 
1139 , errores $=698$; respuestas elaboradas aciertos $=257$; errores $=318$ ) . Este fenómeno afecta tanto a enunciados verdaderos como a enunciados falsos.

\section{TABLA II}

Modelo Linear logarítmico. Efectos de las variables Juicio (J) Implicación emocional (I) y Elaboración de las respuestas $(E)$ sobre las respuestas verdaderas

\begin{tabular}{lccccc}
\hline Efecto & G.L. & \multicolumn{2}{c}{$\mathrm{X}^{2}$} & Asoc. Parcial & \multicolumn{2}{c}{$\begin{array}{c}\text { Asoc. Marginal } \\
\text { Probab. }\end{array}$} & \multicolumn{1}{c}{$\mathrm{X}^{2}$} & \begin{tabular}{c} 
Probab. \\
\hline J.
\end{tabular} \\
I. & 1 & 57.62 & 0.0000 & & \\
E & 2 & 12.66 & 0.0018 & & \\
& 1 & 269.53 & 0.0000 & & \\
JI. & 2 & 3.19 & 0.2028 & 4.07 & 0.1309 \\
JE. & 1 & 56.80 & 0.0000 & 57.67 & 0.0000 \\
IE. & 2 & 95.76 & 0.0000 & 96.63 & 0.0000 \\
JIE. & 2 & 10.28 & 0.0058 & & \\
\hline
\end{tabular}

TABLA III

Modelo Linear logarítmico. Efectos de las variables Juicio (J) Implicación emocional (I) y Elaboración de las respuestas (E) sobre las respuestas verdaderas

\begin{tabular}{lccccc}
\hline Efecto & G.L. & \multicolumn{2}{c}{$\mathrm{X}^{2}$} & Psoc. Parcial & \multicolumn{2}{c}{ Asoc. Marginal } \\
\hline J. & 1 & 13.64 & 0.0002 & & \\
I. & 2 & 273.91 & 0.0000 & & \\
E & 1 & 421.91 & 0.0000 & & \\
JI. & 2 & 6.71 & 0.0350 & 7.31 & 0.0259 \\
JE. & 1 & 9.51 & 0.0020 & 10.11 & 0.0015 \\
IE. & 2 & 5.09 & 0.0786 & 5.69 & 0.0582 \\
JIE. & 2 & 14.25 & 0.0008 & & \\
\hline
\end{tabular}

La Implicación emocional en el tema sobre el que versan las preguntas y respuestas afecta al acierto o error en el juicio (baja implicación aciertos $=558$, errores $=464$; media implicación aciertos $=560$, errores $=350$, y alta implicación aciertos $=399$, errores $=202$ ), lo que genera diferencias significativas entre las condiciones de baja implicación frente a media y alta implicación $\mathrm{X}^{2}=9.50, \mathrm{p}=0.05 \mathrm{y} \mathrm{X}^{2}=21.74, \mathrm{p}=0.000$ respectivamente. Como puede comprobarse, cuando se trata de la condición de baja implicación emocional, el número de errores se incrementa considerablemente.

Si como decíamos la elaboración de la respuesta determina que ésta sea más convincente, puede resultar de interés explicativo para los datos obtenidos, revisar las puntuaciones en credibilidad. La significación entre la variable credibilidad y el acierto en el juicio (Pearson $\mathrm{X}^{2}=27.235 ; \mathrm{p}=.0000$ ), nos permite inferir una asociación según la cual la credibilidad de la respuesta está asociada al juicio de los sujetos y por tanto a sus aciertos y errores. No obstante, estos datos, aunque sugestivos son incompletos y se necesitan análisis más específicos que clarifiquen cual de las variables que hemos manipulado, y bajo qué condiciones determina la credibilidad de las respuestas. 


\section{DISCUSION}

Los objetivos de nuestra investigación eran, por una parte, averiguar en qué medida la elaboración de la respuesta influía la precisión en los juicios de verdad y mentira, lo que nos llevó a presentar una hipótesis para contrastar las posturas teóricas de Littlepage y cols., (1985), que apuntan que la elaboración de respuestas falsas dificultaría un adecuado reconocimiento de éstas. Tal presunción se ha visto cumplida también en nuestro trabajo. Así, el que los sujetos puedan prever con antelación lo que quieren decir a los demás, dificulta la detección de mensajes falsos.

Si consideramos que el decir mentiras es una situación activadora que de alguna forma condiciona la expresión del sujeto, podríamos pensar igualmente que la eliminación de dicha activación a través de la preparación de la respuesta modificará la autopresentación que el sujeto haga de sí mismo. Esto vendría dado bien porque el emisor puede controlar los indicadores de activación generados por la situación (Cody y O'Hair, 1983; Miller y cols., 1983; O'Hair y cols., 1981) o bien porque le permita controlar su patrón expresivo general (ver Becerra y Sánchez, 1989) de forma que no presente rupturas que quepan ser interpretables en términos de engaño.

El que la segunda opción sea la correcta podría explicar por qué los efectos de la elaboración de la respuesta se han manifestado tanto para respuestas falsas como para respuestas verdaderas ya que en ambos casos la tendencia de aciertos y errores se invirtió de la condición de no elaboración a la condición de respuesta elaborada. La preparación anticipada de la respuesta homogeniza el patrón expresivo del emisor e incrementa la dificultad para diferenciar si las respuestas son verdaderas o falsas, al contrario de lo que sucede con las respuestas no elaboradas.

En el mismo sentido podríamos pensar que a través de la elaboración de las respuestas, los emisores consiguen ajustar el contenido verbal de lo que dicen construyendo una respuesta más convincente y por tanto más difícil de identificar como falsa (Kraut, 1978). Un apoyo de esto podrían ser los resultados obtenidos del análisis de las puntuaciones de credibilidad. Sin embargo, como hemos dicho, se requieren análisis complementarios que clarifiquen si la elaboración de la respuesta correlaciona directamente con su credibilidad o existen otras variables mediadoras.

Sería interesante explorar qué ocurre cuando las respuestas además de elaboradas tuviesen contenidos poco creibles lo que además nos permitiría ver en que medida la formación de estereotipos y las primeras impresiones de individuos desconocidos pueden modificar los juicios respecto a enunciados verdaderos y falsos.

Por otra parte se ha confirmado la hipótesis planteada en relación con la influencia que el carácter personal o emocional de los temas podría tener en la detección de la mentira. Hemos podido constatar que las respuestas falsas relacionadas con temas más personales en los cuales el sujeto autoinforma haberse sentido más implicado emocionalmente son más acertados. El único caso donde no se dió este efecto fueron las respuestas falsas elaboradas previamente donde la tendencia de errores y aciertos se invierte en relación a las respuestas no elaboradas.

No hemos encontrado en la literatura una manipulación igual a la nuestra respecto a la temática de las mentiras, por lo cual nuestros resultados no 
son directamente comparables a los obtenidos por otros autores en esta cuestión. Sin embargo nuestros resultados apoyarían lo ya sugerido en otros trabajos (Becerra, 1988) en relación a que mentir respecto a temas emocionales genera más activación que mentir respecto a temas no emocionales. Dicha activación afectaría a la conducta expresiva, delatando al emisor, que hasta el momento podría haber intentado ofrecer una imagen determinada, que en este momento ya no puede controlar, excepto si las mentiras referidas a temas personales son preparadas previamente. En este caso, el emisor no tendría que controlar los aspectos verbales a la vez que los no verbales, obteniendo así un mayor control sobre su conducta.

\section{Referencias}

Bi:cli:rra, A.; Sanchez, F. (1989). "Análisis de las variables implicadas en la detección de la mentira». Revista de Psicología Social, 4(2), 167-176.

CODY, M. J. y O'HAIR, H D. (1983). «Nonverbal communication and deception: Differences in deception cues due to gender and communicator dominance» Communication Monographs, 50, $175-192$.

Cody, M. J., Marston, P. J. y Foster, M. (1984). «Paralinguistic and verbal leakage of deception as a function of attempted control and timing of questions». In R. Bostrum (Ed.) Communication yearbook. Beverly Hills: Sage.

DePaulo, B. M., Rosenthal, R. (1979). "Telling lies". Jour. of Pers. and Soc. Psychol. Vol. 37, 1713-1722.

DePaulo, B. M., Zuckerman, M. y Rosinthal, R. (1980). Detecting deception. Modality effects». En L. Wheeler (Ed.) Review of Personality and Social Psychology, 1. Sage: B. $\mathrm{H}$.

DePaulo, B. M., Lanier, K. y Davis; T. (1983). «Detecting the deceit of the motivated liar». Journal of Personality and Social Psychology, 45, 1096-1103.

DePaulo, B. M., Stone, J. I. y Lassiter, G. D. (1985). «Deceiving and detecting». In B. R. Schlenker (Ed.) The self and social life, 373-370. Nueva York: McGraw-Hill.

Ekman, P. (1980). Mistakes when deceiving. Paper presented at the conference on the Clever Hans Phenomenon, Nueva York, Academic of Sciences.

Ekman, P., Friesen, W. V. (1974). «Detecting Deception from the Body or Face». Journal of Personality and Social Psychology. Vol. 29(3) pp. 288-298.

Gorrman, E. (1959). The presentation of self in everyday life. (Trad. cast.) La presentación de la persona en la vida cotidiana. Amorrortu.

Greene, J. O., O'Hair, H. D., Cody, M. J. y Yen, C. (1985). "Planning and control of behavior during deception». Human communication research, 11, 335-364.

Hocking, J. E., Bauchner, J. Kaminski, E. P. \& Miller, G. R. (1979). «Detecting deceptive communnication from verbal, visual, and paralinguistic cues" Human Communnication Research vol. 6, pp. 33-46.

KRAUT, R. E. (1978). "Verbal and nonverbal cues in the perception of lying». Journal of Personality and Social Psychology, 36, 380-391.

Lindskold, S. y Walters, P. (1983). «Categories for acceptability of lies». Journal of Social Psychology, 120, 129-136.

LitTlepage, G. E. y Pineault, M. A. (1985). «Detection of deception of planned and spontaneous communications». Journal of Social Psychology.

McCornack, S. A. y Parks, M. R. (1985). «Deception detection and relationship development: The other side of the trust. In M. L. McLaughlin (Ed.) Comunication yearbook, 9, 337 389, Beverly Hill, Ca: Sage.

Miller, G. R. y Buroon, J. K. (1983). «Factors affecting assessment of witness credibiility». In N. L. Kerrand y R. M. Bray (Eds.) The Psychology of the courtroom, 169-174. N. Y.: Academic.

O'Hair, H. D., Cody, M. J., y McLaughlin, M. L. (1981). «Prepared lies, spontaneous lies, Machiavellianism and nonverbal communication». Human Communication Research, 7, 325-339.

RIGGIO, R. E. y Friedman, H. S. (1983). «Invividual differences and cues to deception» Journal of Personality and Social Psychology, 45, 889-915.

Riggio, R. E. y Tucker, J., y TrockmorTON, D. (1987). "Social skills and deception ability». Personality and Social Psychology Bulletin, 13, 568-577. 
Zuckerman, M.; DeFrank, R. S.; Hall, J. A.; Larrance, D. T. y Rosenthal, R. (1979). "Facial and vocal cues of deception and honesty". Joumal of Experimental Social Psychology, $15,378-396$.

Zuckerman, M.; DePaulo, B. M. y Rosenthal, R. (1981). Verbal and Nonverbal Communication of Deception. En L. Berkovitz, (Ed.). Advances in Experimental Social Psychology. Vol. 14. Academic Press. NY.

Zuckerman, M.; Koestner, R. y Alton, A. O. (1984). «Learning to detect deception». Journal of Personality and Social Psychology, 46, 519-528.

ZuCKi:RmAn, M.; Koestner, R. y COlella, M. J. (1985). «Learning to detect deception from three communication channels". Journal of Nonverbal bebavior, 9(3). 